\title{
Phenomenological Implications of Brane World Scenarios with low Tension
}

\author{
J.A.R. Cembranos, A. Dobado, A. L. Maroto \\ Depto. de Física Teórica, Universidad Complutense de Madrid, 28040 Madrid, Spain
}

\begin{abstract}
The recent proposal of theories with compactified large extra dimensions is reviewed. We pay especial attention to brane world models with low tension where the only relevant degrees of freedom at low energies are the Standard Model (SM) particles and the branons, which are transversal brane oscillations. By using an effective Lagrangian, we study some phenomenological consequences of these scenarios in a model independent way.
\end{abstract}

\section{ARE EXTRA DIMENSIONS REAL OR ARE THEY JUST SCIENCE FICTION?}

Most of the motivations for considering extra dimension have a theoretical origin. In the last thirty years most of the new developments in theoretical high energy physics required the introduction of extra dimensions. Well known examples are modern KaluzaKlein theories (KK), where the isometries of the extra dimensions appear as gauge symmetries, Supersymmetry (SUSY), which in the superfield formulation can be understood as a symmetry involving extra Grassmann dimensions, Supergravity (SUGRA), Superstrings defined consistently only in 10 dimensions and M-theory, which is supposed to be the eleven dimensional theory underlying the five known superstring theories and eleven dimensional SUGRA. One important exception to this rule are the Grand Unified Theories (GUT) but still the most interesting examples from the phenomenological point of view are supersymmetric since they give rise to gauge coupling unification.

The first attempts to extend general relativity to include electromagnetism date back to Theodor Kaluza and Oscar Klein [1]. The discovery of 11D SUGRA produced a revival of the KK ideas in the early 80 's. The first string revolution, triggered by the realization that superstrings can provide a theory of gravitation instead of a theory of strong interactions, the cancelation of the anomalies and the discovery of the heterotic string which opened the door for phenomenology, traslated the interest to 10 dimensions with six dimensional compactified spaces (Calabi-Yau, orbifolds...). The second string revolution of the 90's introduced new ideas such as non-perturbative strings, dualities, branes and the unification of the five known superstring theories in the conjectured Mtheory (these two periods of development of string theory can be reviewed in [2] and [3] respectively).

The main phenomenological problem of the old string theories is that they could not be tested since stringy effects were expected to appear at the Plank scale $M_{P}$ which is of the order of $10^{19} \mathrm{GeV}$. However the new ideas coming from M-theory have inspired new

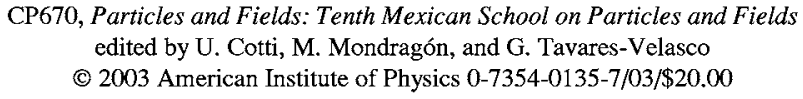


scenarios that could be testable. These scenarios were developed in principle to address the hierarchy problem by putting it in a different setting. The first one was proposed by Arkani-Hamed, Dimopoulos and Dvali (ADD) [4]. The main idea is that our universe is a 3-brane living in a higher $D=4+N$ dimensional space (the bulk space) being the extra dimensions compactified to some small volume (Brane World scenario). In this picture the SM particles are confined to the 3-brane but gravitons can propagate along the whole bulk space. Now the fundamental scale of gravity is not the Planck scale any more but another scale $M_{D}$ which is supposed to be of the order of the electroweak scale in order to solve the hierarchy problem. Then it is possible to find the relation $M_{P}^{2}=V_{N} M_{D}^{2+N}$, where $V_{N}$ is the volume of the compatified extra dimension manifold $B$. Thus the hierarchy between the Planck and the electroweak (TeV) scale is generated by the large volume of the extra dimensions. The typical size $R$ of the extra dimensions ranges from a fraction of $\mathrm{mm}$ for $N=2$ to about $10 \mathrm{fm}$ for $N=6$ (the case $N=1$ is already ruled out by the observations in our solar system). The most interesting property of the ADD scenario (in which we will concentrate in the rest of this work) is that it is compatible with the present experimental data, but at the same time it gives rise to many new phenomena that could be tested in the near future.

There are also scenarios where the scale of the extra dimensions $R$ is of the order of $1 \mathrm{TeV}^{-1}$ [5]. All or some of the SM particles are allowed to propagate along the bulk. This set up is quite appropriate for model building and to deal with gauge coupling unification, SUSY breaking, neutrino spectrum, fermion masses and many other things even if it does not solve the hierarchy problem. In addition there are also models where the hierarchy is generated by the curvature of the extra dimensions as it is the case of the Randall-Sundrum model where the geometry of the space-time is $A d S_{5}$ and thus it cannot be factorized [6].

\section{IS OUR UNIVERSE A 3-BRANE?}

The most obvious consequence of the the Brane World scenario is the modification of the Newton's Law at short distances i.e. distances of the order of $R$. Some recent experiments are trying to test the Newton Law's at the submillimeter scale to explore this possibility (see for instance [7]). $>$ From the point of view of particle physics the main new effects in the ADD scenario are related with the KK mode expansion of the bulk gravitational field

$$
g_{\mu v}(x, \vec{y})=\sum_{\vec{k}} g_{\mu v}(x) e^{i \vec{k} \cdot \vec{y} / R}
$$

where a toroidal compactification has been assumed for simplicity, $\vec{y}$ represents the $N$ extra dimension coordinates and $\vec{k}$ is a $N$ dimensional vector with components $k^{a}=0,1,2, \ldots$. Therefore a bulk graviton can be understood as a KK tower of four dimensional massive gravitons with masses of the order of $k / R$ with $k$ being any natural number (for the $N=1$ case) so that the distance in the mass spectrum between two consecutive KK gravitons is of the order of $1 / R$. This means in particular that the KK graviton spectrum can be considered as approximately continuous for large extra dimensions. In principle we expect two kinds of effects from the KK graviton tower, namely 
graviton production and virtual effects on other particle production or observables (see for instance [8] and references therein). The rates for the different processes can be computed by linearizing the bulk gravitational field and by coupling the graviton field to the SM energy momentum tensor $T_{S M}^{\mu \nu}$. Then expanding the gravitational field in terms of the KK modes one finds the corresponding Feynman rules. As an example one could consider the process $e^{+} e^{-} \rightarrow \gamma+G$ where $G$ stands for any KK graviton. To compute the total cross section one must sum (integrate for large extra dimensions) over all the KK gravitons. The result is

$$
\sigma_{K K} \simeq \frac{1}{M_{P}^{2}}(\sqrt{s} R)^{N} \simeq \frac{1}{M_{D}^{2}}\left(\frac{\sqrt{s}}{M_{D}}\right)^{N}
$$

Note that the total cross section is suppressed by powers of $M_{D}$ which is supposed to be of the order of $1 \mathrm{TeV}$ instead of powers of $M_{P}$ which would make the cross section completely negligible. The signature of these events would be one single photon plus missing energy with a continuous spectrum. Virtual effects can be taken into account by considering the $\mathrm{KK}$ tower propagator

$$
\sum_{\vec{k}} \frac{1}{-p^{2}+k^{2} / R^{2}}
$$

This propagator produce divergencies for more than one extra dimension even at the tree level that require regularization by introducing some ultraviolet cutoff. This is one of facts that has given rise to the development of the so called deconstructing [9] or aliphatic [10] idea where the extra dimensions are latticed.

\section{BRANE FLUCTUATIONS}

However there is a more physical way to deal with the above problem. So far we have asssumed that the world brane is completely rigid, i.e. it has infinite tension. But rigid objects do not exist in relativistic theories. When brane fluctuations are taken into account two new effects appear. First of all we have to introduce new fields which represent the position of the brane in the bulk space $\left(x^{\mu}, y^{a}=\pi^{a}(x)\right)$. These fields are the Goldstone bosons (GB) corresponding to the spontaneous symmetry breaking (SSB) of the translational invariance produced by the presence of the brane (branons). When the effect of the branons is taken into account it is possible to show that the coupling of the SM particles on the brane to any bulk field is exponentially suppressed by a factor $\exp \left(-M_{K K}^{2} M_{D}^{2} /\left(8 \pi^{2} f^{4}\right)\right)$, where $M_{K K}$ is the KK mode mass and $\tau=f^{4}$ is the brane tension [11]. This effect solves the above mentioned divergency problem. The other important consequence of this effect is that in the case where $f \ll M_{D}$ i.e., when the brane tension scale is much smaller than the fundamental scale of gravity (in the $D$ dimensional space), the KK modes decouple from the SM particles on the brane. The conclusion is that for flexible enough branes the only relevant degrees of freedom at low energies in the ADD scenario are the SM particles and the branons. As other GB, 
branons are expected to be nearly massless ${ }^{1}$ and weakly interacting at low energies, and their interactions can be described by an effective lagrangian. For homogeneous extra dimension compact space $B$, this effective lagragian has the structure of a non-linear sigma model where the coset space is just (is isomorphic to) the space $B$ [12]. In addition the branon couple by pairs to the energy-momentum tensor of the SM, $T_{S M}^{\mu \nu}$ through the tensor $\partial_{\mu} \pi^{a} \partial_{v} \pi^{a}$ quite in the same way as gravitons do. Thus it is possible to obtain the different Feynman rules and amplitudes for branon production starting from SM particles in terms of the SM parameters, the brane tension scale $f$, the number of branons $n(n=N$ for homogeneous spaces $B)$ and the possible branon mass $M$ ([13]). The typical signature for branon production would be missing energy with continuous spectrum, but in this case the missing energy spectrum is continuous due to the fact that branons are produced in pairs. In addition by studying branon production one is probing the brane tension scale $f$ instead of the fundamental gravitation scale $M_{D}$ which is probed in graviton production. In the following we will consider the branon phenomenology in the ADD scenario with a flexible brane $f \ll M_{D}$ in order to set some bounds on the brane tension and the branon mass from particle physics, astrophysics and cosmology.

\section{BOUNDS FROM Z AND $W^{ \pm}$DECAYS}

In the Standard Model, the full decay width of the $\mathrm{Z}$ boson has three types of contributions, coming from charged leptons, hadrons and neutrinos respectively. The third one corresponds to the so called invisible width, since neutrinos escape without producing any signal in the detectors. In principle, light branons $\left(M<M_{Z} / 2\right)$ being stable and chargeless particles, could also give rise to invisible decays of $\mathrm{Z}$ and therefore to deviations with respect to the SM predictions.

The precision measurements done mostly at LEP-I set stringent limits on the $\mathrm{Z}$ invisible decay width, which can be translated into strong bounds on the brane tension $f$ and the branon mass. Thus, at the $95 \%$ confidence level, the variation of the $\mathrm{Z}$ invisible width due to decays into branons $\Gamma_{Z}^{b}(n, f, M)$ cannot be larger than $2.0 \mathrm{MeV}$ [14].

We use the results from [13] for the first order contribution from branons to this width, which is given by the decay of $Z$ into two branons and two neutrinos: $Z \rightarrow \bar{v} \vee \pi \pi$. Imposing the LEP-I limit on this decay width we get exclusion plots in the $f-M$ plane (see Figure 1, left for models with a single branon). For $n$ massless branons, the bound on $f$ reads: $f>11.9 n^{1 / 8} \mathrm{GeV}$.

Concerning the $W^{ \pm}$decay, there are mainly two new channels contributing to this process. First, the decay of $W^{ \pm}$into two branons and two leptons: $W^{-} \rightarrow l^{-} \bar{v} \pi \pi$. Such leptons can be an electron and an electron antineutrino for the $W^{-}$decay, their antiparticles for $W^{+}$, or the analogue pairs of leptons in the rest of families. Second, we have the $W^{ \pm}$decay into two branons and a quark-antiquark pair: $W \rightarrow q \bar{q} \pi \pi$. In principle there should be also a channel including gluons in the final state, however in the SM, such channel is suppressed by a coefficient $\alpha_{S}\left(M_{W}\right) / \pi=0.04$ and for that

\footnotetext{
${ }^{1}$ Some branon mass $M$ could be expected from explicit symmetry breaking effects as it happens with pions, which are the GB related to the SSB of the chiral symmetry of strong interactions
} 
reason we will also ignore it in the present case. In any case, the result we will obtain will be an strict lower bound to the true decay width into branons.

The bounds on the contribution from new physics to the $W^{ \pm}$width $\Gamma_{W}^{b}<240 \mathrm{MeV}$, are weaker than the $\mathrm{Z}$ case. This limit translates into the following one for massless branons: $f>6.9 n^{1 / 8} \mathrm{GeV}$. For a single branon, we have plotted the exclusion region for the variables $f$ and $M$ in Figure 1 (left) [13].

\section{BOUNDS FROM DIRECT SEARCHES}

The differential cross section of the process: $e^{+} e^{-} \rightarrow \pi \pi \gamma$ including the two photon polarizations reads [13]:

$$
\begin{aligned}
\frac{d \sigma_{\gamma}}{d x d(\cos \theta)}= & \alpha_{E M}^{2} \frac{2 s\left(s(1-x)-4 M^{2}\right)^{2} n}{61440 f^{8} \pi^{2}} \sqrt{1-\frac{4 M^{2}}{s(1-x)}} \\
& {\left[x\left(3-3 x+2 x^{2}\right)-x^{3} \sin ^{2} \theta+\frac{2(1-x)\left(1+(1-x)^{2}\right)}{x \sin ^{2} \theta}\right] }
\end{aligned}
$$

Such expression could be used in direct searches of branons in colliders. However, we have calculated the contribution to the total cross section of processes involving a single photon in the final state. The total cross section can be calculated analytically, although we will not show it here since the final expression is quite long. However the result for massless branons $(\mathrm{M}=0)$ is much simpler:

$$
\sigma_{\gamma}(M=0)=\frac{n A s^{3}}{f^{8}} .
$$

where the constant depends on the detection limits. For the LEP-II experiments we get $A=4.54 \times 10^{-7}$. This has allowed us to obtain new bounds on $f$ assuming no observation at LEP-II: $f \geq 138 n^{1 / 8} \mathrm{GeV}$.

The results in the massive case for one branon assuming no observation is plotted in Figure 1 (right). Thus the interior area limited by the bound curve is potentially excluded by the LEP-II experiment.

Following similar steps to the single-photon case, we compute the limits from the single- $Z$ channel [13]. In this case the total cross section is obtained by summing over the three $\mathrm{Z}$ polarizations. The result for massless branons is now: $f \geq 52 n^{1 / 8} \mathrm{GeV}$, and the exclusion plot can be found in Figure 1 (right). The excluded area limited by the bound curve is smaller than in the single photon analysis. This is an expected result since the $\mathrm{Z}$ coupling to the electron field is smaller than that of the photon, and the $\mathrm{Z}$ mass restricts the avalaible phase space. In fact, this restricts the search only to branons with masses: $M \leq\left(\sqrt{s}-M_{Z}\right) / 2 \simeq 57 \mathrm{GeV}$. In the single-photon channel however, the kinematical range is larger $M \leq \sqrt{s} / 2 \simeq 103 \mathrm{GeV}$. In future colliders, working at very high centre-of-mass energy, the $Z$ mass could be neglected $\sqrt{s} \gg M_{Z}$, and both processes will allow us to get similar bounds. 

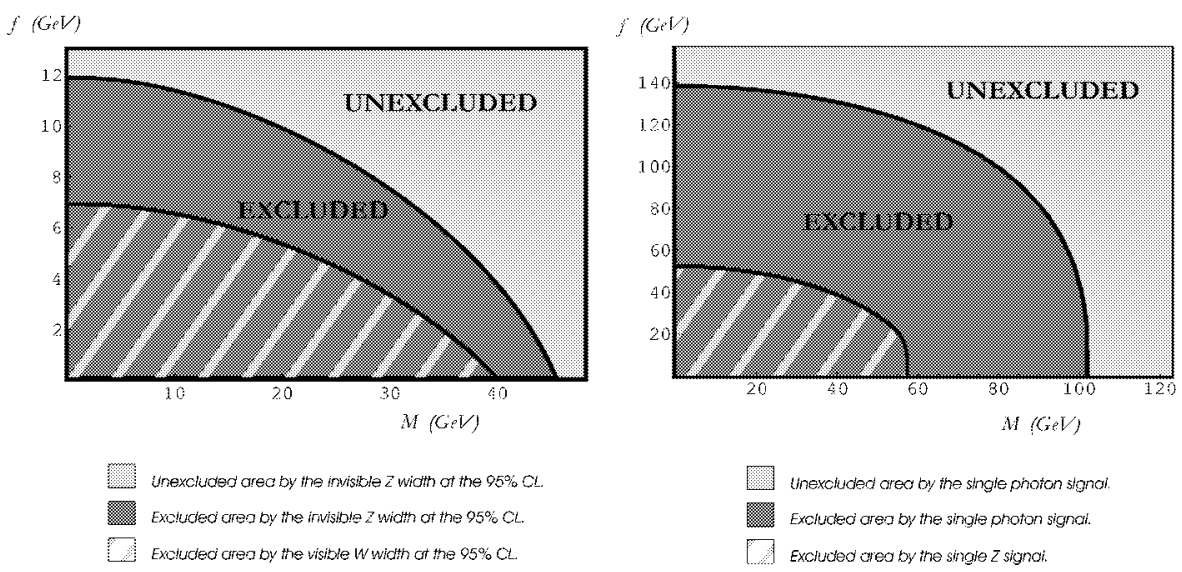

FIGURE 1. Left: exclusion plot in the $f-M$ plane for single-branon models from LEP-I data. The dark area is excluded by $Z$ invisible width data and the striped region is excluded by the visible $W^{ \pm}$decay. Right: exclusion regions in the $f-M$ plane coming from single-photon events (dark region) and single-Z processes (striped area), assuming no observation at LEP-II.

\section{PROSPECTS FOR FUTURE LINEAR COLLIDERS}

Several proposals for the construction of $e^{+} e^{-}$linear colliders in the $\mathrm{TeV}$ range are currently under study. The TESLA (TeV Energy Superconducting Linear Accelerator), the NLC (Next Linear Collider) and the JLC (Japanese Linear Collider) are examples of the first generation of these colliders, whereas the CLIC (Compact Linear Collider) would correspond to the second generation. In this section we discuss the sensitivity of these colliders to a hypothetical branon signal.

The physics programme of the new linear collider projects includes the measurement of electroweak parameters with improved precision, such as the invisible $\mathrm{Z}$ width or the $W^{ \pm}$width. However, and since the deviations due to the presence of branons increase dramatically with energy, the largest sensitivity to a branon signal is expected in direct searches like single- $\gamma$ and single- $Z$.

For this purpose the LEP-II study of the previous section is extended to higher centerof-mass energies. It is assumed that, at the time of construction of these accelerators, the theoretical and systematic uncertainties on Standard Model processes will be controlled at the level of the femtobarn. Under this assumption, we have estimated the sensitivity limit by scaling the LEP-II estimate by the expected gain in statistics due to the luminosity improvement.

The critical parameter in the analysis is the center-of-mass energy, $E_{C M}$. In the single photon channel, this leads to limits on $f$ for any branon mass $M<E_{C M} / 2$. The results of a full study in the $(f, M)$ plane and in different experimental contexts are presented in Figure 2. For the single- $Z$, the bounds are less restrictive, and the study is only applicable 
to branon masses below $\left(E_{C M}-M_{Z}\right) / 2$, due to kinematic constraints (see also Figure 2).
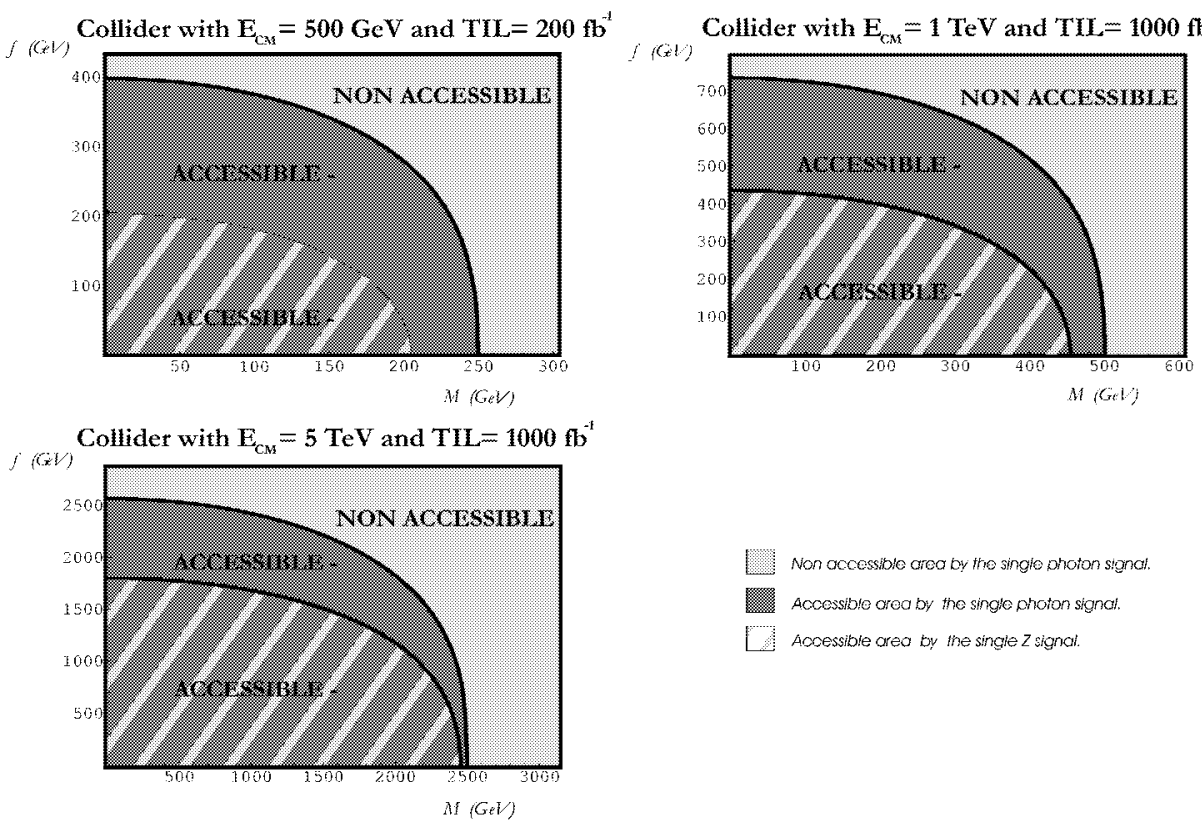

FIGURE 2. Predicted experimental accessible regions in the $f-M$ plane coming from single-photon events and single-Z processes, for future $e^{+} e^{-}$linear colliders with different center-of-mass energies $\left(E_{C M}\right)$ and total integrated luminosities (TIL).

\section{BOUNDS FROM ASTROPHYSICS AND COSMOLOGY}

Since branon effects grow strongly with energy, it is not surprising that the bounds from direct searches that we have just obtained with $(\sqrt{s} \simeq 200 \mathrm{GeV})$ are more constraining than the indirect ones, in which the energy scale is set by the $Z$ mass $\left(M_{Z} \simeq 90 \mathrm{GeV}\right)$. In addition the bounds presented here improve the astrophysical ones for massless branons, coming from energy loss in supernova 1987A, from which $f>10 \mathrm{GeV}$ [15]. For massive branons, the astrophysical limits are much less restrictive (see [16]).

Concerning cosmology, it is interesting to notice that the allowed range of parameters suggests that branons could have weak couplings and large masses. Since they are stable particles, this makes them natural dark matter candidates. In fact, an explicit calculation shows that their relic abundance can be cosmologically relevant and could account for the fraction of one third of the total energy density of the universe in the form of dark matter presently favoured by observations.

On the other hand, the present limits on dark matter abundance can be used also to restrict the parameters space of the theory. The results are complementary to those of colliders. In the latter case, as we have seen, the unexcluded region corresponds to small cross sections (large $f$ ) and/or large branon masses, whereas having cosmological 
branon abundances compatible with observations requires either large cross sections (small $f$ ) or small branon masses. These results will be presented elsewhere [16].

\section{SUMMARY AND CONCLUSIONS}

Brane world scenarios are inspired on modern string (M) theory and offer new insights on many fundamental problems in particle physics. If the fundamental scale of gravity $M_{D}$ is of the order of $1 \mathrm{TeV}$, gravitons could be produced in the next generation of colliders such as the LHC. Detailed computations of the corresponding cross sections require to take into account the recoil effects of the brane. In the flexible brane limit $(f \ll$ $M_{D}$ ) the only relevant modes are the SM particles and the branons. Branon production rates and their cosmological and astrophysical implications can be determined in a model independent way in terms of the brane tension and the branon mass.

Acknowledgements: This work has been partially supported by the DGICYT (Spain) under the project numbers PB98-0782, AEN99-0305, FPA 2000-0956 and BFM20001326. A.D. acknowledges the hospitality of the LBNL Theory Group, where this work was partially done, economical support from the Universidad Complutense del Amo Program and very especially the organizers of the X Mexican School of Particles and Fields for their kind invitation and congratulates Augusto García and Arnulfo Zepeda for their 60th birthay.

\section{REFERENCES}

1. T. Kaluza, Sitzungsberichte of the Prussian Acad. Sci. 966 (1921) O. Klein, Z. Phys. 37 (1926) 895

2. Superstring Theory: M.B. Green, J.H. Schwarz and E. Witten, Cambridge University Press (1987)

3. $\quad$ String Theory: J. Polchinski, Cambridge University Press (1998)

4. N. Arkani-Hamed, S. Dimopoulos and G. Dvali, Phys. Lett. B429, 263 (1998)

N. Arkani-Hamed, S. Dimopoulos and G. Dvali, Phys. Rev. D59, 086004 (1999)

5. I. Antoniadis, Phys. Lett. B246 377 (1990)

6. L. Randall and R. Sundrum, Phys. Rev. Lett. 83, 3370 (1999) and Phys. Rev. Lett. 83, 4690 (1999)

7. C.D. Hoyle et al. EOT-WASH Group Collaboration Phys. Rev. Lett. 86, 1418 (2001)

8. J. Hewett and M. Spiropulu, hep-ph/0205106

9. N. Arkani-Hamed, A.G. Cohen and H. Georgi,Phys. Rev. Lett. 86, 4757 (2001)

10. H. Cheng, C.T. Hill, S. Pokorski and J. Wang, Phys. Rev. D64, 065007 (2001)

11. M. Bando, T. Kugo, T. Noguchi and K. Yoshioka, Phys. Rev. Lett. 83, 3601 (1999)

12. A. Dobado and A.L. Maroto Nucl. Phys. B592, 203 (2001)

13. J. Alcaraz, J.A.R. Cembranos, A. Dobado and A.L. Maroto, hep-ph/0212269

14. A Combination of Preliminary Electroweak Measurements and Constraints on the Standard Model. The LEP Collaborations, the LEP Electroweak Working Group and the SLD Heavy Flavour Group, LEPEWWG/2002-01, hep-ex/0112021.

15. T. Kugo and K. Yoshioka, Nucl. Phys. B594, 301(2001)

16. J.A.R. Cembranos, A. Dobado and A.L. Maroto. Work in preparation. 
Copyright $\odot 2003$ EBSCO Publishing 
Copyright of AIP Conference Proceedings is the property of American Institute of Physics and its content may not be copied or emailed to multiple sites or posted to a listserv without the copyright holder's express written permission. However, users may print, download, or email articles for individual use. 